\title{
Virtual monochromatic dual-energy CT reconstructions improve detection of cerebral infarct in patients with suspicion of stroke
}

\author{
Fasco van Ommen ${ }^{1,2}$ (D) Jan Willem Dankbaar ${ }^{2} \cdot$ Guangming Zhu ${ }^{1} \cdot$ Dylan N. Wolman ${ }^{1} \cdot$ Jeremy J. Heit ${ }^{1}$. \\ Frans Kauw $^{1,2}$. Edwin Bennink ${ }^{2,3} \cdot$ Hugo W. A. M. de Jong ${ }^{2,3} \cdot$ Max Wintermark $^{1}$
}

Received: 25 May 2020 / Accepted: 5 July 2020 / Published online: 29 July 2020

(C) The Author(s) 2020

\begin{abstract}
Purpose Early infarcts are hard to diagnose on non-contrast head CT. Dual-energy CT (DECT) may potentially increase infarct differentiation. The optimal DECT settings for differentiation were identified and evaluated.

Methods One hundred and twenty-five consecutive patients who presented with suspected acute ischemic stroke (AIS) and underwent non-contrast DECT and subsequent DWI were retrospectively identified. The DWI was used as reference standard. First, virtual monochromatic images (VMI) of 25 patients were reconstructed from 40 to $140 \mathrm{keV}$ and scored by two readers for acute infarct. Sensitivity, specificity, positive, and negative predictive values for infarct detection were compared and a subset of VMI energies were selected. Next, for a separate larger cohort of 100 suspected AIS patients, conventional non-contrast CT (NCT) and selected VMI were scored by two readers for the presence and location of infarct. The same statistics for infarct detection were calculated. Infarct location match was compared per vascular territory. Subgroup analyses were dichotomized by time from last-seen-well to CT imaging.

Results A total of 80-90 keV VMI were marginally more sensitive (36.3-37.3\%) than NCT $(32.4 \% ; p>0.680)$, with marginally higher specificity $(92.2-94.4$ vs $91.1 \% ; p>0.509)$ for infarct detection. Location match was superior for VMI compared with NCT (28.7-27.4 vs $19.5 \% ; p<0.010)$. Within $4.5 \mathrm{~h}$ from last-seen-well, $80 \mathrm{keV}$ VMI more accurately detected infarct $(58.0$ vs $54.0 \%)$ and localized infarcts $(27.1 \mathrm{vs} 11.9 \% ; p=0.004)$ than NCT, whereas after $4.5 \mathrm{~h}, 90 \mathrm{keV}$ VMI was more accurate $(69.3 \mathrm{vs} 66.3 \%)$. Conclusion Non-contrast 80-90 keV VMI best differentiates normal from infarcted brain parenchyma.
\end{abstract}

Keywords Stroke $\cdot$ Non-contrast CT $\cdot$ Dual-energy CT $\cdot$ Virtual monochromatic images

Electronic supplementary material The online version of this article (https://doi.org/10.1007/s00234-020-02492-y) contains supplementary material, which is available to authorized users.

Fasco van Ommen

F.vanOmmen@umcutrecht.nl

Jan Willem Dankbaar

J.W.Dankbaar@umcutrecht.nl

Guangming Zhu

zhugmdc@stanford.edu

Dylan N. Wolman

dwolman1@stanford.edu

Jeremy J. Heit

jheit@stanford.edu

Frans Kauw

F.Kauw-3@umcutrecht.nl
Edwin Bennink

H.E.Bennink-2@umcutrecht.nl

Hugo W. A. M. de Jong

H.W.A.M.deJong@umcutrecht.nl

Max Wintermark

mwinterm@stanford.edu

1 Department of Neuroradiology, Stanford University Medical Center, Palo Alto, CA, USA

2 Department of Radiology and Nuclear Medicine, University Medical Center Utrecht, E01.132, P.O. Box 85500, 3508 GA Utrecht, the Netherlands

3 Image Sciences Institute, University Medical Center Utrecht, Utrecht, the Netherlands 


$\begin{array}{ll}\text { Abbreviations } & \\ \text { AIS } & \text { Acute ischemic stroke } \\ \text { DECT } & \text { Dual-energy CT } \\ \text { VMI } & \text { Virtual monochromatic images } \\ \text { NCT } & \text { Conventional non-contrast head CT } \\ \text { mASPECTS } & \text { Modified ASPECTS } \\ \text { HU } & \text { Hounsfield units } \\ \text { WL } & \text { Window level } \\ \text { WW } & \text { Window width } \\ \text { PPV } & \text { Positive predictive value } \\ \text { NPV } & \text { Negative predictive value } \\ \text { IQR } & \text { Interquartile range }\end{array}$

\section{Introduction}

Non-contrast CT is the mainstay in the initial evaluation of patients with suspicion of acute ischemic stroke (AIS) [1]. However, the sensitivity of non-contrast CT is limited for the detection of acute brain infarct [2]. The reference standard for infarct detection is MRI with diffusion-weighted imaging (DWI) [3, 4]. DWI allows for detection of cytotoxic edema within infarcted tissue with high sensitivity, while CT is limited to detecting subtle changes in water content between infarcted and normal brain parenchyma.

Dual-energy CT (DECT) advances CT imaging by improving upon conventional non-contrast head CT (NCT) by acquiring data with two separate energy spectra, which allows improved contrast resolution, reduced image noise and beamhardening artifacts, and spectral separation of constituent materials at equivalent dose [5, 6]. Virtual monochromatic CT images (VMI) can be derived from source DECT data [7-9], and reflect the tissue properties of a scan acquired at a single specific monochromatic energy level. Leveraging different monoenergetic reconstructions may help accentuate differences in energy-dependent attenuation differences between similar materials, e.g., normal brain tissue and ischemic/ edematous brain tissue. DECT VMI have demonstrated improved contrast-to-noise profiles and reduced beam-hardening artifact relative to NCT [10-15]. The potential of DECT for the visualization of brain edema has been investigated in earlier studies [16-18]. In these studies, elaborate reconstruction methods were required, whereas VMI is a standard derivative of DECT imaging and broadly applicable to any vendor's software. The use of VMI in the detection of cerebral infarct has not been established. We sought to identify the noncontrast VMI energy which best differentiates normal from infarcted brain parenchyma, and to determine if this VMI can more sensitively and specifically identify infarct in patients with suspected AIS compared with NCT.

\section{Materials and methods}

\section{Patients}

This study was approved by the Stanford University Institutional Review Board which waived the need for informed consent, and data collection complied with the Health Insurance Portability and Accountability Act. We retrospectively enrolled consecutive patients between October 13, 2018, and April 18, 2019, with suspected AIS who underwent non-contrast DECT and subsequent MRI with DWI within $48 \mathrm{~h}$. Inclusion criteria were as follows: patient age $>18$ years and presentation within $24 \mathrm{~h}$ of symptom onset. Exclusion criteria were as follows: technical failure of DECT, significant metal artifact limiting interpretation, or corrupted DWI. Baseline clinical data was collected, including age, sex, presentation National Institutes of Health Stroke Scale (NIHSS), time since last known well, time to initial DECT imaging, and time to subsequent MRI.

\section{Imaging protocol}

All patients were imaged using a dual-source Somatom Flash CT scanner (Siemens Healthineers, Erlangen, Germany). Noncontrast dual-energy CT protocols are dose neutral with respect to single-energy acquisitions at our institution. The volume CT dose index (CTDIvol) for each scan was $59.8 \mathrm{mGy}$, and was based on a 16-cm International Electrochemical Commission (IEC) head dosimetry phantom. The scan parameters were as follows: Tube A, $80 \mathrm{kVp}$ and $640 \mathrm{mAs}$; Tube B, $140 \mathrm{kVp}$ with a tin filter and $320 \mathrm{mAs}$, beam collimation of $40 \times 0.6 \mathrm{~mm}$, a 1.0 -s rotation time, matrix size $512 \times 512$, and a pitch of 1.0 . Images were reconstructed at $3 \mathrm{~mm}$ using a medium smoothing Q34s kernel. Non-contrast DECT VMI images were reconstructed using the Monoenergetic+ software module in Syngo Via (Siemens Healthineers, Erlangen, Germany).

\section{Study design}

Reference standard MRI (DWI) was obtained within $48 \mathrm{~h}$ of each patient's index CT and was reviewed by a single neurointerventional radiologist with 7 years of experience (JJH). DWI imaging parameters included the following: TR $6000 \mathrm{~ms}$, TE $78.2 \mathrm{~ms}$, b-values 0 and 1000, flip angle $90^{\circ}$, and 5-mm slice thickness. Each MRI was scored on a binary scale for presence of cerebral infarct, defined as focal parenchymal restricted diffusion. Cerebral infarcts were binned by location using a modified Alberta Stroke Program Early CT Score (mASPECTS) $[19,20]$, in which additional regions corresponding to the posterior circulation (thalamus, superficial PCA, brainstem, and cerebellum) and anterior cerebral artery territory (A1 anteriorly, and A2 posteriorly) are added. The A1 territory is anatomically bounded as the inferior ACA territory, while the A2 territory was bounded by the superior ACA territory. 
Phase 1 of this study was used as an initial selection step to identify the VMI reconstruction energies best suited for infarct detection. Phase 2 compared these identified VMI reconstructions against NCT for the detection of cerebral infarct and qualitative infarct localization to identify VMI energies best for differentiation between normal and infarcted brain tissue.

\section{Phase 1 design}

In the first phase of the study, VMI were reconstructed at $10 \mathrm{keV}$ increments from 40 to $140 \mathrm{keV}$. VMI reconstructions were randomized and reviewed by a neuroradiologist and a neurologist (JWD and GZ, with 12 and 19 years of experience in radiology, respectively, and both more than 10 years of experience in stroke imaging) who were blinded to the reconstruction type, but did have CT indication information. Each reader reviewed a full axial image stack for each series with fixed window level and width settings. As attenuation values (HU) vary with tube voltage [21], the fixed window level (WL) for each reconstruction energy was visually identified by a neuroradiologist (MW) and set to maintain similar attenuation uniformity between series. The settings were as follows: $40-50 \mathrm{keV}$ (WL 50), 60-70 keV (WL 45), 80-110 keV and NCT (WL 40), and 120-140 keV (WL 35). Window width (WW) was set to $50 \mathrm{HU}$ for all reconstructions. Reviewers evaluated for the presence of acute cerebral infarct, and localized infarct foci using the mASPECTS system. Acute infarct was defined as a focal loss of gray-white matter differentiation, focal edema, or hypoattenuation without volume loss. Discrepant reviews were resolved by consensus. A number of VMI reconstructions, who were the most sensitive and specific for the detection of cerebral infarct relative to the reference standard MRI with DWI, were selected and used for comparison with NCT and used to identify the VMI energies that best differentiated normal from infarcted tissue.

\section{Phase 2 design}

In the second phase, two different independent reviewers scored a separate, larger cohort of patients presenting with suspicion for AIS. Each examination was reconstructed as a NCT (WL/WW 40/50) and the most sensitive and specific VMI energies identified in phase $1(60-90 \mathrm{keV})$, and were presented in a randomized, blinded fashion using the same fixed window and level settings as described in phase 1 . An experienced neuroradiologist (JWD) and a neuroradiology fellow (DNW) scored each examination for evidence of acute infarct. Subgroup analyses of patients imaged in the early window ( $\leq$ $4.5 \mathrm{~h}$ after last-seen-well) and those presenting in the late window ( $>4.5 \mathrm{~h}$ after last-seen-well) were performed to assess for time-dependent differences in infarct detection. We expect that infarcts in the early window require different improvement in differentiation between similar attenuating tissue (fat, water, and soft tissue), then infarcts in the late window, and might have an influence on the required VMI energy for infarct detection. The volume of cerebral edema increases over time resulting in increased hypoattenuation on CT. At early time points, the contrast between the edema and normal brain tissue is therefore less conspicuous than at later time points. Increasing the contrast between edema and normal brain tissue at different time points may therefore require different energies.

\section{Statistical analysis}

Baseline patient characteristics were compared between phase 1 and 2 using a Mann-Whitney signed rank test for continuous variables and a chi-squared test for discrete variables. In phase 1 , the sensitivity, specificity, positive predictive value (PPV), and negative predictive value (NPV) were calculated for the detection of cerebral infarct for each scored VMI reconstruction relative to the reference standard MRI with DWI. In phase 2, the sensitivity, specificity, PPV, NPV, and accuracy for the detection of cerebral infarct were calculated for the selected VMI reconstruction and the NCT relative to the reference DWI. Sensitivity and specificity were compared between NCT and VMI using the chi-squared test. Regional infarct localization between DWI and CT was calculated as the infarct location match (ILM), which is defined as the percentage of true positive detections of infarct on CT compared with the total of positive detections for infarct on DWI in an anatomical region. ILM was compared between NCT and VMI for the anterior cerebral artery (ACA; A1 and A2), basal ganglia and insular cortex (BG; caudate, lentiform nucleus, internal capsule and insular cortex), posterior circulation (PCA; thalamus, superficial PCA, cerebellum and brainstem), middle cerebral artery at the level of the basal ganglia (sub-MCA; M1, M2 and M3), middle cerebral artery at the level of the ventricles immediately above the basal ganglia (sup-MCA; M4, M5 and M6), and total of all regions combined using McNemar's test. Statistical significance was set at $\alpha=0.05$. To correct for multiple comparisons, a Bonferroni correction was applied. Inter-rater reliability was evaluated using Cohen's Kappa, for which the following interpretations were used: slight $(0.01-0.20)$, fair $(0.21-0.40)$, moderate $(0.41-0.60)$, good $(0.61-0.80)$, near perfect (0.81-0.99), and perfect agreement (1). Subgroup analyses of early and the late window patients were performed using the same methods. Statistical analyses were performed in SPSS (version 25.0, IBM, New York).

\section{Results}

\section{Patient characteristics}

One hundred and twenty-five consecutive patients suspected of AIS were included (median age 65.0; IQR 51.8-80.3 years; $48 \%$ female). In phase 1, 25 patients were included (median age 67.0; IQR 54.5-75.3 years; $56 \%$ female), while in phase 2 , 
Table 1 Patient characteristics of patients in phase 1 and phase 2 of the study

\begin{tabular}{llll}
\hline Characteristics & Phase 1 $(n=25)$ & Phase 2 $(n=96)$ & $P$ value \\
\hline General & & & \\
Sex, male:female & $11: 14$ & $51: 45$ & 0.416 \\
Age (years), median (IQR) & $67.0(54.5-75.3)$ & $63.0(52.5-81.0)$ & 1.000 \\
Presentation NIHSS, median (IQR) & $4(1-11)$ & $5(2-13)$ & 0.700 \\
Platelets, median (IQR) & $201(167-261)$ & $222(185-275)$ & 0.700 \\
Cerebral infarct on DWI, $n(\%)$ & $12(48.0)$ & $51(53.1)$ & 0.648 \\
Time since last-seen-well (hours), median (IQR) & $5.0(1.4-12.0)$ & $4.2(1.9-12.0)$ & 1.000 \\
Time CT to MR (hours), median (IQR) & $5.0(3.1-11.0)$ & $5.2(3.2-12.7)$ & 0.700 \\
IV tPA administered, $n(\%)$ & $4(16.0)$ & $12(12.5)$ & 0.222 \\
Stroke risk factors & & & \\
Coronary artery disease (CAD), $n(\%)$ & $4(16.0)$ & $13(13.5)$ & 0.753 \\
Atrium fibrillation, $n(\%)$ & $3(12.0)$ & $12(12.5)$ & 0.946 \\
Diabetes, $n(\%)$ & $7(28.0)$ & $23(24.0)$ & 0.677 \\
Hypertension, $n(\%)$ & $15(60.0)$ & $55(57.3)$ & 0.807 \\
Hyperlipemia, $n(\%)$ & $12(48.0)$ & $38(39.6)$ & 0.447 \\
Prior cerebrovascular incident, $n(\%)$ & $3(12.0)$ & $28(29.2)$ & 0.080 \\
Prior intracranial hemorrhage, $n(\%)$ & $1(4.0)$ & $10(10.4)$ & 0.320 \\
Smoker, $n(\%)$ & $3(12.0)$ & $25(26.0)$ & 0.138 \\
Antiplatelet or anticoagulant, $n(\%)$ & $12(48.0)$ & $47(49.0)$ & 0.932 \\
\hline
\end{tabular}

a total of 96 patients (median age 63; IQR 52.5-81.0 years; $46.9 \%$ female) were included after exclusion of 4 patients for metallic artifact (1 CT case) and corrupted DWI images (3 MRI cases). Phase 1 patients had a median presenting NIHSS of 4 (IQR 1-11), while phase 2 patients had a median presentation NIHSS of 5 (IQR 2-12). Baseline patient demographics are summarized in Table 1. There were no significant differences in baseline patient characteristics between the groups.

\section{Phase 1: initial selection VMI energies for infarct detection}

Acute cerebral infarct was identified in 12/25 (48\%) patients by DWI. Sensitivity, specificity, PPV, and NPV of each VMI reconstruction from 40 to $140 \mathrm{keV}$ for the detection of infarct relative to the reference DWI are shown in Fig. 1. The interobserver variability range for the VMI reconstructions was $0.34-0.75$. The $60-120 \mathrm{keV}$ reconstructions showed the highest
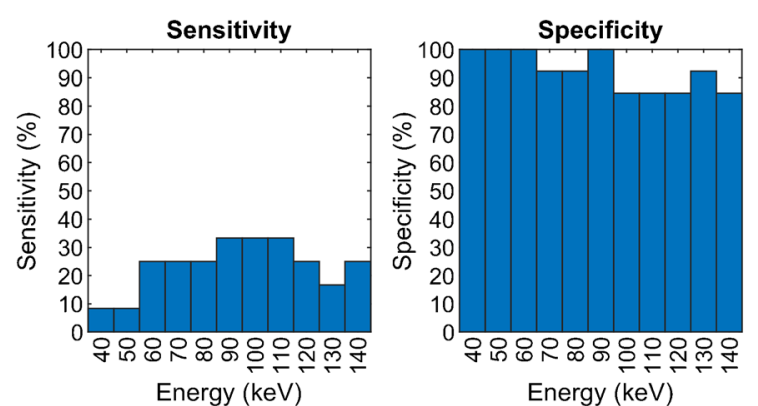

sensitivities (25.0-33.3\%), but 60-90 keV also had a high specificity (92.3-100.0\%), PPV (75.0-100.0\%), and NPV (57.1$61.9 \%$ ) with a moderate inter-reader agreement (0.41-0.45). Sensitivity was lowest for the 40, 50 and $130 \mathrm{keV}$ VMI reconstructions (8.3-16.7\%), whereas the 120 and $140 \mathrm{keV}$ demonstrated lower specificity versus the $60-90 \mathrm{keV}$ reconstructions (84.6\% vs 92.3-100\%). Figure 2 illustrates typical images the observers reviewed.

VMI reconstructions from 60 to $90 \mathrm{keV}$ were the most sensitive and specific for the detection of cerebral infarct, and were chosen for further evaluation in phase 2 .

\section{Phase 2: comparison of VMI with conventional CT}

\section{Infarct detection}

DWI identified 51/96 (53\%) patients with acute infarction. The sensitivity, specificity, PPV, NPV, and accuracy for the
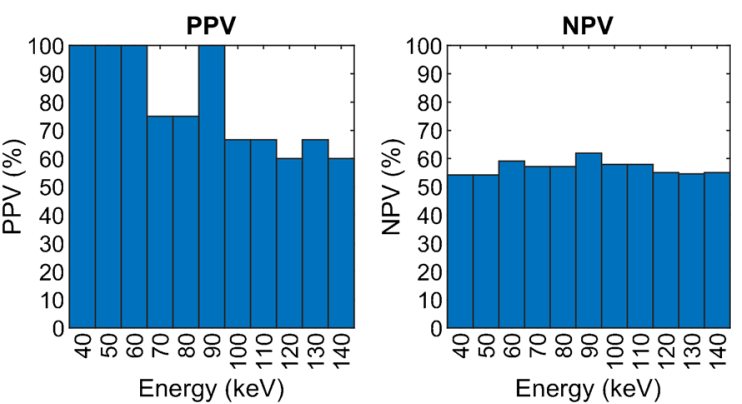

Fig. 1 The sensitivity, specificity, positive predictive value, and negative predictive value of the consensus scoring of the different VMI reconstructions for detection of infarct 


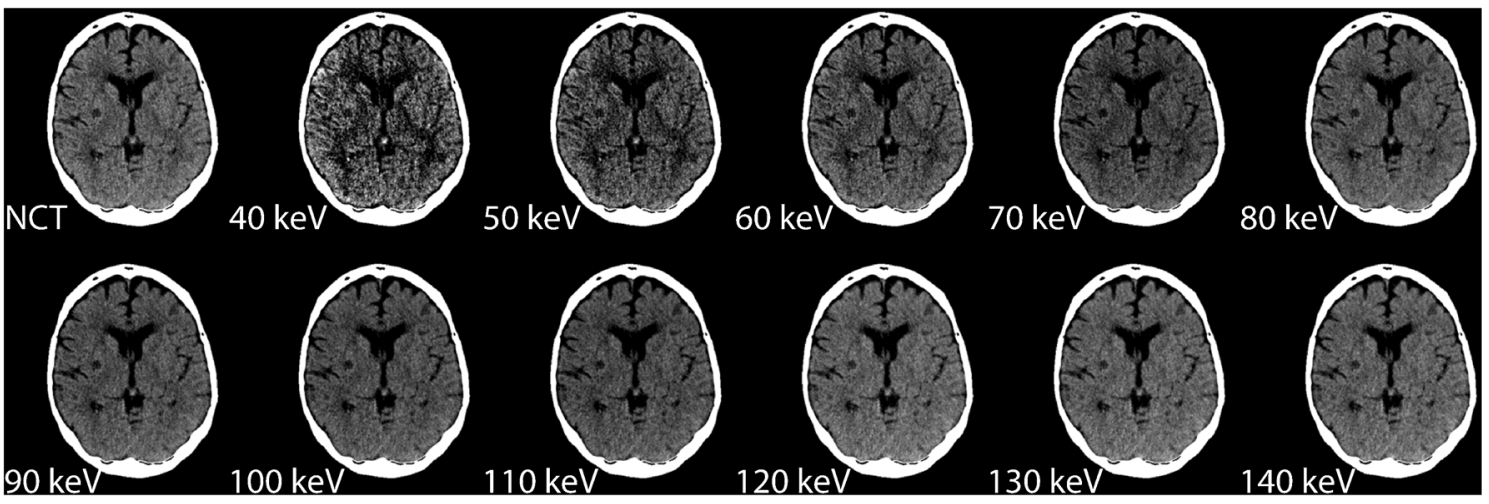

Fig. 2 Example patient of VMI and conventional reconstructions. The NCT has a WL/WW of 40/50, 40, and $50 \mathrm{keV}$ (WL/WW, 50/50), 60 and 70 keV (WL/WW, 45/50), 80, 90, 100, and $110 \mathrm{keV}$ (WL/WW, 40/50), and 120, 130, and $140 \mathrm{keV}$ (WL/WW, 35/50)

detection of infarct for all included VMI reconstructions and the NCT are shown in Table 2. The VMI reconstructions (70$90 \mathrm{keV}$ ) had higher mean sensitivity relative to NCT, even though not significant (36.0-37.3 vs NCT $32.4 \%$; $p>0.680)$. VMI reconstructed at 80 and $90 \mathrm{keV}$ demonstrated higher sensitivity $(36.0,37.3$ vs NCT $32.4 \%$; $p>0.680)$, specificity $(92.2,94.4$ vs NCT 91.1\%; $p>0.509)$, PPV $(84.0,89.1$ vs NCT $82.0 \%)$, NPV $(55.7,56.6$ vs NCT $54.2 \%)$, and accuracy $(62.0,63.5$ vs NCT $59.9 \%)$ compared with the NCT, where $90 \mathrm{keV}$ performed best. Inter-rater reliability for all reconstructions was good (0.64-0.78). Figure 3 illustrates the difference in conspicuity of infarct between VMI and NCT in comparison with DWI.

\section{Infarct per territory}

Pooled ILM of NCT and VMI are shown in Table 3. NCT and VMI were unable to match the location of infarct in the anterior cerebral artery to DWI. ILM-NCT was higher in the posterior circulation (11.1-13.3 vs $15.5 \%$ ), though not significant. Other regions (sub-MCA, sup-MCA, and basal ganglia) were higher for 80 and $90 \mathrm{keV}$ VMI compared to NCT, and these differences were significant in the middle cerebral artery at the level of the basal ganglia (sub-MCA; 29.6-33.3 vs NCT $7.4 \%$; $p$ <.015). ILM for 80 and $90 \mathrm{keV}$ reconstructions for all regions was significantly higher compared to NCT (28.7 and $27.4 \%$ vs. $19.5 \% ; p<0.010)$. The inter-reader reliability was near perfect for all CT reconstructions $(0.86-0.88)$.

\section{Infarct in the early and late windows}

Fifty patients were scanned within the early window (median time 1.9, IQR 0.8-3.0 h). Among these patients, $25(50.0 \%)$ had DWI-positive acute cerebral infarct. Forty-six patients were scanned within the late window (median time 12.0, IQR 6.3-17.9 h). Among these patients, 26 (56.5\%) had DWI-positive acute cerebral infarct.

For early window patients, VMI reconstructions at $80 \mathrm{keV}$ showed the highest mean sensitivity (26.0\%), whereas NCT had $18.0 \%$ sensitivity. The difference, however, is not significant $(p=0.499)$. In addition, $80 \mathrm{keV}$ VMI showed similar or higher specificity (90.0 vs $90.0 \%$; $p=1.000)$, PPV (73.8 vs $64.3 \%)$ NPV (54.8 vs $52.3 \%)$, and accuracy (58.0 vs $54.0 \%$ ) relative to NCT. A good inter-rater reliability was seen for all reconstructions (0.66-0.79). Patients in the late window showed higher sensitivity for $90 \mathrm{keV}$ compared with NCT $(50.0 \mathrm{vs}$ $46.2 \%$; $p=0.786)$. Specificity was higher for $90 \mathrm{keV}$ compared with NCT (95.0 vs $92.5 \% ; p=0.747)$. PPV (93.3 vs $90.6 \%)$, NPV (0.59.3 vs $56.9 \%$ ), and accuracy (69.6 vs $66.3 \%$ ) are also higher for $90 \mathrm{keV}$ compared with NCT. NCT and VMI showed good inter-rater reliability $(0.61-$

Table 2 Infarct detection

\begin{tabular}{|c|c|c|c|c|c|c|}
\hline & Sensitivity (R1, R2) & Specificity (R1, R2) & PPV (R1, R2) & NPV (R1, R2) & Accuracy (R1, R2) & $\operatorname{IRR}(95 \% \mathrm{CI})$ \\
\hline NCT & $32.4(33.3,31.4)$ & $91.1(97.8,84.4)$ & $82.0(94.4,69.6)$ & $54.2(56.4,52.1)$ & $59.9(63.5,56.3)$ & $0.64(0.51-0.77)$ \\
\hline $60 \mathrm{keV}$ & $30.4(25.5,35.3)$ & $85.6(95.6,75.6)$ & $74.4(86.7,62.1)$ & $51.9(53.1,50.7)$ & $56.3(58.3,54.2)$ & $0.71(0.60-0.83)$ \\
\hline $70 \mathrm{keV}$ & $36.0(33.3,38.7)$ & $86.7(93.3,80.0)$ & $77.0(85.0,69.0)$ & $54.5(55.3,53.7)$ & $59.9(61.5,58.3)$ & $0.65(0.52-0.78)$ \\
\hline $80 \mathrm{keV}$ & $36.0(38.7,33.3)$ & $92.2(97.8,86.7)$ & $84.0(95.2,72.7)$ & $55.7(58.7,52.7)$ & $62.0(66.7,57.3)$ & $0.73(0.61-0.85)$ \\
\hline $90 \mathrm{keV}$ & $37.3(37.3,37.3)$ & $94.4(100.0,88.9)$ & $89.1(100.0,78.3)$ & $56.6(58.4,54.8)$ & $63.5(66.7,60.4)$ & $0.78(0.67-0.89)$ \\
\hline
\end{tabular}

Infarct detection of VMI and conventional CT (NCT) shown in sensitivity (\%), specificity (\%), PPV (\%), NPV (\%), accuracy (\%), and inter-reader reliability (IRR), with 95\% confidence interval (CI). The sensitivity, specificity, PPV, NPV, and accuracy of each individual observer (R1, R2) are provided parenthetically after the pooled value 
Fig. 3 Example case of a patient demonstrating the increased conspicuity of an acute infarct on the 80 and $90 \mathrm{keV} \mathrm{VMI} \mathrm{in}$ comparison to NCT, 60 and 70 $\mathrm{keV}$ and corresponding diffusion restriction on subsequent MRI DWI confirmed the territory of infarct. Red arrows indicate the outlining of the infarcted area. The CT reconstructions have the same WL/WW, 40/30

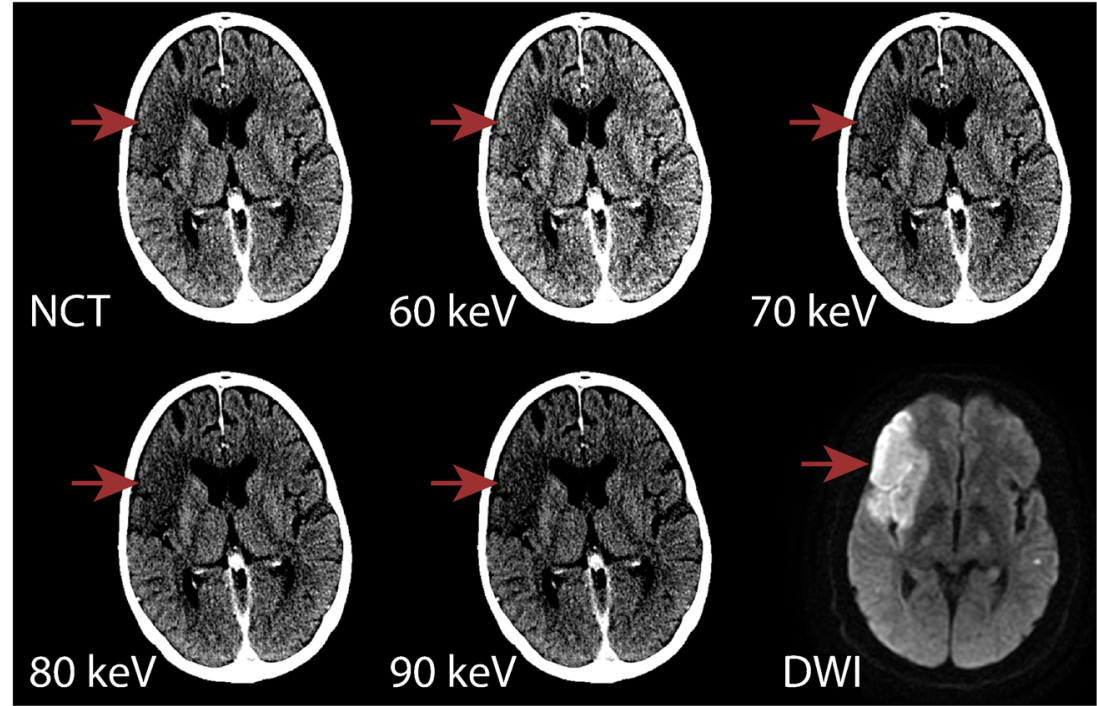

0.77). Results of other VMI in early and late window are shown in Table 1 of supplementary materials.

Pooled ILM of NCT and VMI in the early- and late-windows are shown in Table 4. In the early window, location matching on VMI at $80 \mathrm{keV}$ was similar or better than NCT in all regions (ACA, $0.0 \%$ vs. $0.0 \%$, sub-MCA; $36.4 \%$ vs. $0.0 \%$, sup-MCA; $43.8 \%$ vs. $25.0 \%$, BG; $36.4 \%$ vs. $18.2 \%$ and PCA; $5.9 \%$ vs. $5.9 \%$ ). The increase, however, was not significant in any of the regions. ILM of all regions of 80 $\mathrm{keV}$ VMI $(27.1 \%)$ was significantly higher $(p=0.012)$ compared to total ILM of NCT $(11.9 \%)$. The inter-reader reliability was near perfect for all CT reconstructions (0.83-0.85). In the late window, VMI at $90 \mathrm{keV}$ outperformed NCT for all territories (ACA, $0.0 \%$ vs. $0.0 \%$, sub-MCA; $37.5 \%$ vs. $12.5 \%$, supMCA; $39.4 \%$ vs. $36.4 \%$ and BG; $40.9 \%$ vs. $22.7 \%$ ), except in the posterior circulation (PCA, $17.9 \%$ vs. $21.4 \%$ ). The differences were not significant. The ILM of all regions of $90 \mathrm{keV}$ VMI (31.4\%) was significantly higher compared to NCTILM of all regions $(23.8 \% ; p=0.031)$. The inter-reader reliability was near perfect for all CT reconstructions (0.88-0.89).

\section{Discussion}

In this study, VMI reconstructions at $90 \mathrm{keV}$ showed higher sensitivity $(36.3 \%)$ and specificity $(94.4 \%)$ for the detection of acute cerebral infarct in AIS patients than NCT $(32.4 \%$ and $91.1 \%$, respectively). This VMI energy is concordant with prior reports suggesting that optimal VMI for maximum contrast resolution is between 60 and $100 \mathrm{keV}$ in adults depending on the DECT approach $[10,14,22]$. Pomerantz et al. [10] suggested $65-75 \mathrm{keV}$ for overall parenchymal contrast resolution, with agreement by Neuhaus et al. [14] at $65 \mathrm{keV}$ for gray-white differentiation, whereas Yoshida et al. [22] suggested that $99 \mathrm{keV}$ offered superior evaluation of supratentorial acute infarct. Yoshida et al., however, only used confirmed infarct

Table 3 ILM comparison for anterior cerebral artery, middle cerebral artery, basal ganglia, and posterior circulation

\begin{tabular}{lcccccc}
\hline Region & DWI $(n)$ & \multicolumn{2}{l}{ ILM (\%) (95\% CI) } \\
\cline { 3 - 6 } & \multicolumn{7}{l}{ NCT } & $60 \mathrm{keV}$ & $70 \mathrm{keV}$ & $80 \mathrm{keV}$ & $90 \mathrm{keV}$ \\
\hline ACA & 10 & $0.0(0.0-12.5)$ & $0.0(0.0-12.5)$ & $0.0(0.0-12.5)$ & $0.0(0.0-12.5)$ & $0.0(0.0-12.5)$ \\
Sub-MCA & 27 & $7.4(2.1-23.4)$ & $3.7(0.7-18.3)$ & $18.5(8.2-36.7)$ & $33.3(18.6-52.2) *$ & $29.6(15.9-48.5) *$ \\
Sup-MCA & 49 & $32.7(21.2-46.6)$ & $22.4(13.0-35.9)$ & $36.7(24.7-50.7)$ & $38.8(26.4-52.8)$ & $38.8(26.4-52.8)$ \\
BG & 33 & $21.2(10.7-37.8)$ & $21.2(10.7-37.8)$ & $39.4(24.7-56.3)$ & $39.4(24.7-56.3)$ & $36.4(22.2-53.4)$ \\
PCA & 45 & $15.6(7.8-28.8)$ & $13.3(6.3-26.2)$ & $11.1(4.8-23.5)$ & $13.3(6.3-26.2)$ & $13.3(6.3-26.2)$ \\
All & 164 & $19.5(14.2-26.2)$ & $15.2(10.5-21.5)$ & $25.0(19.0-32.2)$ & $28.7(22.3-36.0) *$ & $27.4(21.2-34.7) *$ \\
IRR (95\% CI) & & $0.87(0.86-0.89)$ & $0.88(0.86-0.90)$ & $0.87(0.85-0.89)$ & $0.86(0.84-0.88)$ & $0.86(0.84-0.88)$ \\
\hline
\end{tabular}

Pooled ILM of VMI and NCT with 95\% confidence interval (CI) are presented and compared. If the difference between NCT and VMI is significant, it is highlighted with an asterisk. In addition, inter-reader reliability (IRR) with $95 \%$ confidence interval is shown for each CT reconstruction. Regions: $A C A$, anterior cerebral artery (A1 and A2); $B G$, basal ganglia (caudate, lentiform nucleus, internal capsule and insular cortex); $P C A$, posterior circulation (thalamus, superficial PCA, cerebellum and brainstem); sub-MCA, middle cerebral artery at level basal ganglia (M1, M2, and M3); and sup-MCA, middle cerebral artery at the level of the ventricles immediately above the basal ganglia (M4, M5, and M6) 
Table 4 ILM comparison for anterior cerebral artery, middle cerebral artery, basal ganglia, and posterior circulation as a function of time

\begin{tabular}{|c|c|c|c|c|c|c|}
\hline \multirow[t]{2}{*}{ Region } & \multirow[t]{2}{*}{ DWI $(n)$} & \multicolumn{5}{|l|}{$\operatorname{ILM}(\%)(95 \% \mathrm{CI})$} \\
\hline & & NCT & $60 \mathrm{keV}$ & $70 \mathrm{keV}$ & $80 \mathrm{keV}$ & $90 \mathrm{keV}$ \\
\hline \multicolumn{7}{|l|}{$\leq 4.5 \mathrm{~h}$} \\
\hline ACA & 4 & $0.0(0.0-49.0)$ & $0.0(0.0-49.0)$ & $0.0(0.0-49.0)$ & $0.0(0.0-49.0)$ & $0.0(0.0-49.0)$ \\
\hline Sub-MCA & 11 & $0.0(0.0-25.9)$ & $0.0(0.0-25.9)$ & $27.3(9.7-56.6)$ & $36.4(15.2-64.6)$ & $18.2(5.1-47.7)$ \\
\hline Sup-MCA & 16 & $25.0(10.2-49.5)$ & $12.5(3.5-36.0)$ & $37.5(18.5-61.4)$ & $43.8(23.1-66.8)$ & $37.5(18.5-61.4)$ \\
\hline $\mathrm{BG}$ & 11 & $18.2(5.1-47.7)$ & $0.0(0.0-25.9)$ & $9.1(1.6-37.7)$ & $36.4(15.2-64.6)$ & $27.3(9.7-56.6)$ \\
\hline PCA & 17 & $5.9(1.1-27.0)$ & $5.9(1.1-27.0)$ & $5.9(1.1-27.0)$ & $5.9(1.1-27.0)$ & $5.9(1.1-27.0)$ \\
\hline All & 59 & $11.9(5.9-22.5)$ & $5.1(1.7-13.9)$ & $18.6(10.7-30.4)$ & $27.1(17.4-39.6)^{*}$ & $20.3(12.0-32.3)$ \\
\hline IRR (95\% CI) & & $0.85(0.82-0.88)$ & $0.85(0.83-0.88)$ & $0.85(0.82-0.87)$ & $0.83(0.80-0.86)$ & $0.83(0.80-0.85)$ \\
\hline \multicolumn{7}{|l|}{$>4.5 \mathrm{~h}$} \\
\hline ACA & 6 & $0.0(0.0-39.0)$ & $0.0(0.0-39.0)$ & $0.0(0.0-39.0)$ & $0.0(0.0-39.0)$ & $0.0(0.0-39.0)$ \\
\hline Sub-MCA & 16 & $12.5(3.5-36.0)$ & $6.3(1.1-28.3)$ & $12.5(3.5-36.0)$ & $31.3(14.2-55.6)$ & $37.5(18.5-61.4)$ \\
\hline Sup-MCA & 33 & $36.4(22.2-53.4)$ & $27.3(15.1-44.2)$ & $36.4(22.2-53.4)$ & $36.4(22.2-53.4)$ & $39.4(24.7-56.3)$ \\
\hline BG & 22 & $22.7(10.1-43.4)$ & $31.8(16.4-52.7)$ & $54.5(34.7-73.1)$ & $40.9(23.3-61.3)$ & $40.9(23.3-61.3)$ \\
\hline PCA & 28 & $21.4(10.2-39.5)$ & $17.9(7.9-35.6)$ & $14.3(5.7-31.5)^{*}$ & $17.9(7.9-35.6)$ & $17.9(7.9-35.6)$ \\
\hline All & 105 & $23.8(16.7-32.8)$ & $21.0(14.3-29.7)$ & $28.6(20.8-37.9)$ & $29.5(21.6-38.8)$ & $31.4(23.3-40.8) *$ \\
\hline IRR $(95 \%$ CI) & & $0.89(0.86-0.91)$ & $0.89(0.86-0.92)$ & $0.88(0.85-0.90)$ & $0.88(0.85-0.90)$ & $0.88(0.85-0.90)$ \\
\hline
\end{tabular}

Pooled ILM of VMI and NCT with 95\% confidence interval (CI) are compared in the early- and late-windows. If the difference between NCT and VMI is significant, it is highlighted with an asterisk. In addition, inter-reader reliability (IRR) with $95 \%$ confidence interval is shown for each CT reconstruction. Regions: $A C A$, anterior cerebral artery (A1 and A2); $B G$, basal ganglia (caudate, lentiform nucleus, internal capsule and insular cortex); $P C A$, posterior circulation (thalamus, superficial PCA, cerebellum and brainstem); sub-MCA, middle cerebral artery at level basal ganglia (M1, M2, and M3), and sup$M C A$, middle cerebral artery at the level of the ventricles immediately above the basal ganglia (M4, M5 and M6)

patients; we included AIS patients. By comparing our CT findings with DWI, we were able to identify the best VMI energies for differentiating between normal and infarcted tissue. Additionally, VMI reconstructions at $90 \mathrm{keV}$ also had the highest NPV (56.6 vs $54.2 \%$ ). As CT is often used as a screening modality in cases where there is concern for AIS, improvements in the sensitivity and NPV offered by VMI may lend confidence to the neuroradiologist in excluding cerebral infarct, particularly if MRI is contraindicated or unavailable.

Subgroup analyses of patients imaged $\leq 4.5 \mathrm{~h}$ and $>4.5 \mathrm{~h}$ from last-seen-well were performed as infarct conspicuity changes with increasing edema in the maturing lesion. Analysis of patients presenting $\leq 4.5 \mathrm{~h}$ from last known well demonstrated a small improvement in sensitivity (26.0 vs 18.0 ) and NPV (54.8 vs $52.3 \%$ ), and similar specificity $(90.0 \%)$ for the detection of acute cerebral infarct using VMI at $80 \mathrm{keV}$ as compared with NCT. Given that patients presenting within this window are considered eligible for intravenous thrombolytic therapy, even small improvements in infarct detection may increase clinical and therapeutic certainty [23].

Infarct localization relative to reference standard DWI was assessed using ILM for each pre-defined cerebral regions. Within the middle cerebral artery territories (sub-MCA and sup-MCA), a non-significant trend towards superior VMIILM at $80-90 \mathrm{keV}$ was observed relative to NCT-ILM.
However, overall VMI-ILM showed a significant improvement with $80-90 \mathrm{keV}$ in comparison with total NCT-ILM (28.7, 27.4 vs $19.5 \%$ ), which suggests that VMI may significantly increase the accuracy of infarct localization. Given regional improvement in infarct detection of VMI seen at $80 \mathrm{keV}$ in the early window within the anterior circulation, assessment of VMI in an anterior large-vessel occlusion population is warranted. To determine if infarct core may be better estimated with VMI than with NCT and could be used for early window treatment decisions [24], in our study, we see a clear trend towards a significant increase in ILM using VMI; however, our study was underpowered given the number of territories surveyed, and further testing in a larger cohort is warranted.

Relative to NCT, VMI reconstructions at $80-90 \mathrm{keV}$ showed a significant improvement of acute infarct detection. This improvement is most likely due to the qualitatively demonstrated improved soft tissue contrast. This improved contrast therefore increases small inherent attenuation differences between gray matter, white matter, and edematous tissue compared with NCT. We hypothesize that the increase in contrast is due to increased differences between attenuation of gray and white matter and to the lower noise levels in 80 and $90 \mathrm{keV}$ VMI $[15,22]$. The range of best VMI energies is $80-90 \mathrm{keV}$, and we recommend individual institutions to test their own optima, but 
$90 \mathrm{keV}$ generally has a slightly better sensitivity and specificity for infarct detection compared with $80 \mathrm{keV}$. Infarct location detection is slightly better with $80 \mathrm{keV}$. However, if dual-energy CT is unavailable, similar results are unlikely to be obtained by modifying single-energy CT protocols to an average energy of $90 \mathrm{keV}$, as this will involve an increase in peak tube voltage and a substantial decrease in exposure to maintain a similar dose level. This increase in $\mathrm{kVp}$ will result in a decrease in contrast, because there will be a large number of photons with an energy higher than $90 \mathrm{keV}$ which will not attenuate in tissue.

Our study has several limitations. First, our retrospective study design may introduce bias. The small sample size may limit our statistical analysis, particularly for analysis of ILM. Second, our sensitivity analysis may be artificially increased as the imaging reviewers were aware that the testing population was enriched for patients with cerebral infarct. Third, a recognition bias can be assumed, resulting in an underestimation of the differences between the reconstruction types. We, however, blinded our reviewers to reconstruction type, CT reconstructions were presented in a random fashion, and the large amount of CT reconstructions in the comparison ensured that no single energy level was favored over the other. Lastly, we investigated the applicability of VMI using a single type of DECT acquisition (dual-source CT scanner). DECT acquisitions, VMI techniques, and reconstruction algorithms vary between manufacturers and types of DECT scanners. As a result, monochromatic energy levels cannot be reproduced between manufacturers or post-processing algorithms, thereby limiting study generalizability and indicating that different institutions may need to determine VMI optima independently [25].

In conclusion, use of virtual monochromatic images at 80 $90 \mathrm{keV}$ with non-contrast dual-energy CT results in a modest improvement of sensitivity and specificity for the detection of acute infarcts in suspected ischemic stroke patients relative to conventional non-contrast CT.

Funding information This research has been made possible by the Dutch Heart Foundation and Technology Foundation STW, as part of their joint strategic research program (project number 14732): earlier recognition of cardiovascular diseases.

\section{Compliance with ethical standards}

Conflict of interest The authors declare that they have no conflict of interest.

Ethical approval All procedures performed in the studies involving human participants were in accordance with the ethical standards of the institutional and/or national research committee and with the 1964 Helsinki Declaration and its later amendments or comparable ethical standards.

Informed consent Need for informed consent was waived by our Institutional Review Board.
Open Access This article is licensed under a Creative Commons Attribution 4.0 International License, which permits use, sharing, adaptation, distribution and reproduction in any medium or format, as long as you give appropriate credit to the original author(s) and the source, provide a link to the Creative Commons licence, and indicate if changes were made. The images or other third party material in this article are included in the article's Creative Commons licence, unless indicated otherwise in a credit line to the material. If material is not included in the article's Creative Commons licence and your intended use is not permitted by statutory regulation or exceeds the permitted use, you will need to obtain permission directly from the copyright holder. To view a copy of this licence, visit http://creativecommons.org/licenses/by/4.0/.

\section{References}

1. Wintermark M, Sanelli PC, Albers GW, Bello J, Derdeyn C, Hetts SW, Johnson MH, Kidwell C, Lev MH, Liebeskind DS, Rowley H, Schaefer PW, Sunshine JL, Zaharchuk G, Meltzer CC (2013) Imaging recommendations for acute stroke and transient ischemic attack patients: a joint statement by the American Society of Neuroradiology, the American College of Radiology, and the Society of NeuroInterventional Surgery. Am J Neuroradiol 34: E117-E127. https://doi.org/10.3174/ajnr.A3690

2. Wardlaw JM, Mielke O (2005) Early signs of brain infarction at CT: observer reliability and outcome after thrombolytic treatment systematic review. Radiology 235:444-453. https://doi.org/10. 1148/radiol.2352040262

3. Muir KW, Buchan A, von Kummer R, Rother J, Baron J-C (2006) Imaging of acute stroke. Lancet Neurol 5:755-768. https://doi.org/ 10.1016/S1474-4422(06)70545-2

4. Merino JG, Warach S (2010) Imaging of acute stroke. Nat Rev Neurol 6:560-571. https://doi.org/10.1038/nrneurol.2010.129

5. McCollough CH, Leng S, Yu L, Fletcher JG (2015) Dual- and multi-energy CT: principles, technical approaches, and clinical applications. Radiology 276:637-653. https://doi.org/10.1148/radiol. 2015142631

6. Wolman DN, Patel BP, Wintermark M, Heit JJ (2018) Dual-energy computed tomography applications in neurointervention. J Comput Assist Tomogr 42:831-839. https://doi.org/10.1097/RCT. 0000000000000779

7. Wellenberg RHH, Boomsma MF, van Osch JAC, Vlassenbroek A, Milles J, Edens MA, Streekstra GJ, Slump CH, Maas M (2017) Quantifying metal artefact reduction using virtual monochromatic dual-layer detector spectral CT imaging in unilateral and bilateral total hip prostheses. Eur J Radiol 88:61-70. https://doi.org/10. 1016/j.ejrad.2017.01.002

8. Yu L, Christner JA, Leng S, Wang J, Fletcher JG, McCollough CH (2011) Virtual monochromatic imaging in dual-source dual-energy CT: radiation dose and image quality. Med Phys 38:6371-6379. https://doi.org/10.1118/1.3658568

9. Zhou C, Zhao YE, Luo S, Shi H, Li L, Zheng L et al (2011) Monoenergetic imaging of dual-energy CT reduces artifacts from implanted metal orthopedic devices in patients with factures. Acad Radiol 18:1252-1257. https://doi.org/10.1016/j.acra.2011.05.009

10. Pomerantz SR, Kamalian S, Zhang D, Gupta R, Rapalino O, Sahani DV, Lev MH (2013) Virtual monochromatic reconstruction of dual-energy unenhanced head CT at $65-75 \mathrm{keV}$ maximizes image quality compared with conventional polychromatic CT. Radiology 266:318-325. https://doi.org/10.1148/radiol.12111604

11. Lam S, Gupta R, Levental M, Yu E, Curtin HD, Forghani R (2015) Optimal virtual monochromatic images for evaluation of normal 
tissues and head and neck cancer using dual-energy CT. Am J Neuroradiol 36:1518-1524. https://doi.org/10.3174/ajnr.A4314

12. Hwang WD, Mossa-Basha M, Andre JB, Hippe DS, Culbertson S, Anzai Y (2016) Qualitative comparison of noncontrast head dualenergy computed tomography using rapid voltage switching technique and conventional computed tomography. J Comput Assist Tomogr 40:320-325. https://doi.org/10.1097/RCT. 0000000000000350

13. Zhao X-M, Wang M, Wu R-Z, Dharaiya E, Feng F, Li M-L, You H, Wang Y, Wang YN, Jin ZY (2018) Dual-layer spectral detector CT monoenergetic reconstruction improves image quality of noncontrast cerebral CT as compared with conventional single energy CT. Eur J Radiol 103:131-138. https://doi.org/10.1016/j.ejrad. 2018.04.015

14. Neuhaus V, Abdullayev N, Große Hokamp N, Pahn G, Kabbasch C, Mpotsaris A, Maintz D, Borggrefe J (2017) Improvement of image quality in unenhanced dual-layer $\mathrm{CT}$ of the head using virtual monoenergetic images compared with polyenergetic single-energy CT. Investig Radiol 00:1-476. https://doi.org/10.1097/RLI. 0000000000000367

15. Park J, Choi YH, Cheon JE, Kim WS, Kim IO, Pak SY, Krauss B (2017) Advanced virtual monochromatic reconstruction of dualenergy unenhanced brain computed tomography in children: comparison of image quality against standard mono-energetic images and conventional polychromatic computed tomography. Pediatr Radiol 47:1648-1658. https://doi.org/10.1007/s00247-017-3908-8

16. Taguchi K, Itoh T, Fuld MK, Fournie E, Lee O, Noguchi K (2018) "X-map 2.0" for edema signal enhancement for acute ischemic stroke using non-contrast-enhanced dual-energy computed tomography. Investig Radiol 53:432-439. https://doi.org/10.1097/RLI. 0000000000000461

17. Grams AE, Djurdjevic T, Rehwald R, Schiestl T, Dazinger F, Steiger R, Knoflach M, Gizewski ER, Glodny B (2018) Improved visualisation of early cerebral infarctions after endovascular stroke therapy using dual-energy computed tomography oedema maps. Eur Radiol 28:4534-4541. https://doi.org/10.1007/s00330-0185449-4

18. Mohammed MF, Marais O, Min A, Ferguson D, Jalal S, Khosa F, O Keeffe M, O Connell T, Schmiedeskamp H, Krauss B, Rohr A, Nicolaou S (2018) Unenhanced dual-energy computed tomography. Investig Radiol 53:63-69. https://doi.org/10.1097/RLI. 0000000000000413

19. Barber PA, Demchuk AM, Zhang J, Buchan AM (2000) Validity and reliability of a quantitative computed tomography score in predicting outcome of hyperacute stroke before thrombolytic therapy. Lancet 355:1670-1674. https://doi.org/10.1016/S01406736(00)02237-6

20. Puetz V, Sylaja PN, Coutts SB, Hill MD, Dzialowski I, Mueller P, Becker U, Urban G, O’Reilly C, Barber PA, Sharma P, Goyal M, Gahn G, von Kummer R, Demchuk AM (2008) Extent of hypoattenuation on CT angiography source images predicts functional outcome in patients with basilar artery occlusion. Stroke 39: 2485-2490. https://doi.org/10.1161/STROKEAHA.107.511162

21. Park JE, Choi YH, Cheon JE, Kim WS, Kim IO, Cho HS, Ryu YJ, Kim YJ (2017) Image quality and radiation dose of brain computed tomography in children: effects of decreasing tube voltage from 120 $\mathrm{kVp}$ to $80 \mathrm{kVp}$. Pediatr Radiol 47:710-717. https://doi.org/10. 1007/s00247-017-3799-8

22. Yoshida M, Nakaura $\mathrm{T}$, Tanoue $\mathrm{S}$, Takada $\mathrm{S}$, Inoue $\mathrm{T}$, Uetani $\mathrm{H}$, Harada K, Yamashita Y (2019) Dual-energy computed tomography for evaluating acute brain infarction of middle cerebral artery territories. J Comput Assist Tomogr 43:460-466. https://doi.org/10. 1097/RCT.0000000000000869

23. Powers WJ, Rabinstein AA, Ackerson T, Adeoye OM, Bambakidis NC, Becker K, Biller J, Brown M, Demaerschalk BM, Hoh B, Jauch EC, Kidwell CS, Leslie-Mazwi TM, Ovbiagele B, Scott PA, Sheth KN, Southerland AM, Summers DV, Tirschwell DL, on behalf of the American Heart Association Stroke Council (2019) Guidelines for the early management of patients with acute ischemic stroke: 2019 update to the 2018 guidelines for the early management of acute ischemic stroke: a guideline for healthcare professionals from the American Heart Association/American stroke. Stroke 50:344-418. https://doi.org/10.1161/STR. 0000000000000211

24. Powers WJ, Rabinstein AA, Ackerson T, Adeoye OM, Bambakidis NC, Becker K, Biller J, Brown M, Demaerschalk BM, Hoh B, Jauch EC, Kidwell CS, Leslie-Mazwi TM, Ovbiagele B, Scott PA, Sheth KN, Southerland AM, Summers DV, Tirschwell DL, American Heart Association Stroke Council (2018) 2018 guidelines for the early management of patients with acute ischemic stroke: a guideline for healthcare professionals from the American Heart Association/American Stroke Association. Stroke 49:e46e110. https://doi.org/10.1161/STR.0000000000000158

25. Potter CA, Sodickson AD (2016) Dual-energy CT in emergency neuroimaging: added value and novel applications. RadioGraphics 36:2186-2198. https://doi.org/10.1148/rg.2016160069

Publisher's note Springer Nature remains neutral with regard to jurisdictional claims in published maps and institutional affiliations. 